\title{
Single Pulse Illumination of Multi-Layer Photoacoustic Holograms for Patterned Ultrasound Field Generation
}

\author{
Michael D. Brown, Eleanor Martin, Ben T. Cox and Bradley E. Treeby \\ Department of Medical Physics and Biomedical Engineering, University College London \\ 2-10 Stephenson Way, London, NW1 2HE \\ Email: michael.brown.13@ucl.ac.uk
}

\begin{abstract}
A new method for the creation of patterned, focused, optically generated acoustic fields using a single optical pulse is introduced. This utilises multi-layer 'holograms' composed of several spatially separate absorbing layers. Each layer is individually patterned so as to focus at a set of targeted points. To create the patterns, a ray-tracing model was implemented to calculate the impulse response of pixels within each absorbing layer to a set of targeted points. An optimisation approach was then used to find the optimal pattern for each layer to create a field evenly focused at each of the target points. The method was validated using both numerical simulations and acoustic field measurements. It was demonstrated that a $3 \times 3$ array of acoustic foci could be generated from a 3-layer hologram using a single laser pulse.
\end{abstract}

\section{INTRODUCTION}

The optical generation of ultrasound (OGUS), in which a thin optically absorbing layer emits ultrasound following optical irradiation, has in recent years attracted increasing attention for certain applications in biomedical ultrasound. This is partly due to the development of more efficient photoacoustic transmitters, increasing the pressures which can be achieved [1]. However, spatially steering and focusing OGUS fields currently poses a number of significant difficulties.

One approach proposed to overcome this limitation is to modulate the spatial pattern and temporal shape of the light incident on a planar absorber. This enables control of both the acoustic spectra and the spatial pattern of the initial pressure distribution. These combined allow the use of photoacoustic holography to control where the resulting acoustic field constructively and destructively interferes [2]. Several previous works have explored this approach demonstrating the generation of a number of novel acoustic fields [3, 4]. However, at present several expensive Q-switch laser sources are required to generate the pressure amplitudes necessary for biomedical applications.

An alternative to temporal and spatial modulation of the incident light is to shape the surface on which the absorber is deposited. In the simplest case, an absorber can be deposited on a concave lens. This results in the generation of a single high pressure foci at the focus of the lens when it is illuminated by a pulsed laser [5]. Other works have reported the use of an axicon surface for the generation of ultrasonic X-waves [6],

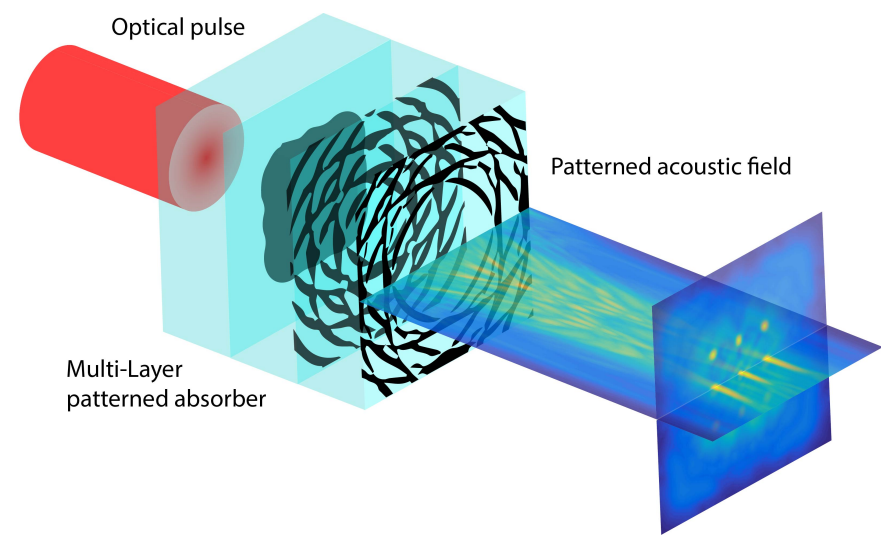

Fig. 1. Illustration of a multi-layered patterned absorber for the generation of patterned OGUS fields. The pattern on each layer and their spacing are tailored such that constructive interference is achieved at the desired focal points.

and the use of a circular ramp for the generation of a helicoidal beam [7].

This work introduces a new approach for the generation of optically generated acoustic fields with multiple foci. This is achieved by creating ' 3 -D holograms'. These are structures made up of several optically absorbing layers each separated by a transparent spacer of a known thickness. Each absorbing layer is patterned so that when it is excited by an optical pulse an acoustic signal is generated only from portions of the layer. The patterns are calculated so that the acoustic signal generated from each layer arrives simultaneously at a set of targeted focal points. This concept is illustrated in Fig. 1. This shows an absorber with 3 discrete layers designed to generate a $3 \times 3$ array of acoustic foci in a plane parallel to the absorber.

\section{ABSORBER DESIGN}

\section{A. Overview}

The goal in designing the 3-D hologram is to find a set of binary patterns that, when optically excited, will generate high peak pressures at the target points and minimal pressure elsewhere. Therefore, as a first step in calculating these patterns it is necessary to know the acoustic signal $p(\mathbf{r}, t ; x, y, d)$ generated at a target point at $\mathbf{r}$ at a time $t$, by each source 
pixel $(x, y)$ in each layer $d$, so that the signal generated by a particular set of patterns can be predicted. This is equivalent to needing to know the impulse response of each pixel to each target point.

\section{B. Impulse Response Simulation}

The impulse responses were approximated using a raytracing approach. This was chosen to minimise compute times compared to using a full wave model. The inputs to the ray-tracing model consist of a set of targeted focal points, the boundaries of the hologram relative to the focal points, the position of each absorbing layer, the pixel spacing, and the sound speed and density $\left(c_{h}, \rho_{h}\right)$ of the hologram and $\left(c_{m}, \rho_{m}\right)$ the coupled medium.

The ray-trajectories are selected by evenly sampling between $0-\frac{\pi}{2}$ in the transmitted angle $\theta_{t}$. These angles are then transformed into a set of incident angles $\theta_{i}$ using Snell's law. For each $\theta_{i}$, the number $\mathrm{N}$ of radial angles $\phi$ used is weighted based on the radius of interaction with the surface of the absorber. This is calculated using

$$
N=A h \tan \theta_{i}+B
$$

Here $h$ is the distance from the plane of the foci to the top layer of the absorber, and $A$ and $B$ are constants used to weight the sampling density. The set of values for $\phi$ for each $\theta_{i}$ are chosen using

$$
\phi_{n}=\frac{2 \pi n}{N}+\frac{2 \pi C}{N} .
$$

Here $C$ is a random number between $0-1$ and $n$ is an integer between $1-N$. This ensures the whole range $0-2 \pi$ is sampled. The random factor is introduced to stop systematic sampling errors at certain radial positions.

The propagation of each ray is modelled using Snell's law and the Fresnel equations. Wave spreading is accounted for using an equation first introduced by Young [8]. This gives the pressure $p$ at a particular depth for a point source above a medium change as

$$
p=\frac{T \cos \theta_{i}}{\left(h+d \frac{c_{h}}{c_{m}} \cos \theta_{i} \sec \theta_{t}\right)^{\frac{1}{2}}\left(h+d \frac{c_{h}}{c_{m}} \cos ^{3} \theta_{i} \sec ^{3} \theta_{t}\right)^{\frac{1}{2}}} .
$$

Here $d$ is the vertical distance to the point below the interface, and $\mathrm{T}$ is the transmission coefficient for that incident angle given from the Fresnel equations by

$$
T=\frac{2 \rho_{h} c_{h} \cos \theta_{i}}{\rho_{h} c_{h} \cos \theta_{i}+\rho_{m} c_{m} \cos \theta_{t}}
$$

For each intersection of a ray with a pixel $(x, y, d)$, the time of arrival $t$, the amplitude, and the original trajectory $\left(\theta_{i}, \phi_{i}\right)$ of the ray are stored. As the sampling density of the pixels varies, and wave spreading is already modelled using Eq. (3), the amplitude of rays with similar initial trajectories are averaged.

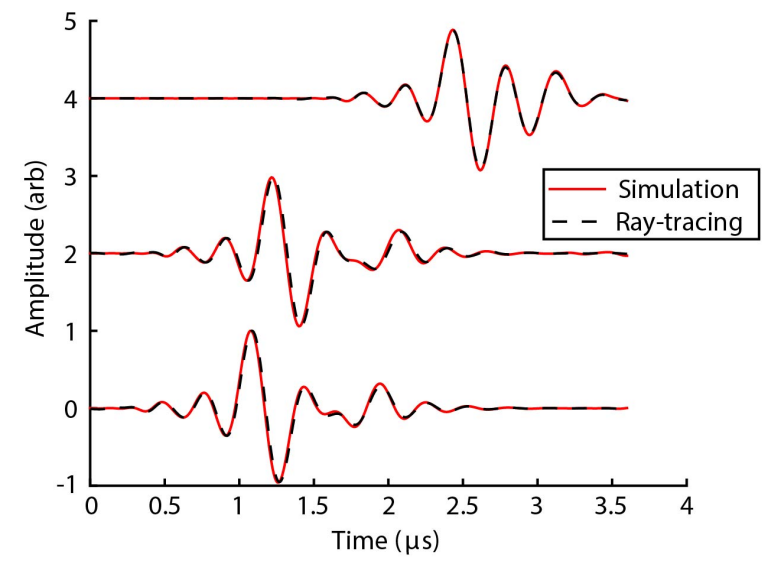

Fig. 2. Comparison of the acoustic response calculated at three different points using the ray-tracing approach (dashed line) and full wave simulation (continuous line). The time series for the ray-tracing and simulation are each normalised to the maximum pressure across all three points for the respective method. At all 3 points good agreement is found over the first $4 \mu$ s.

The output of the ray-tracing is a set of impulse responses $I(\mathbf{r}, t ; x, y, d)$. To get the acoustic signal $p(\mathbf{r}, t ; x, y, d)$ generated by each pixel in an experimental setting these are convolved with the expected signal generated optically from the absorber $s(t)$.

The ray-tracing approximation was validated using the open-source k-Wave toolbox. This uses a k-space pseudospectral model for time domain simulations of acoustic waves [9]. The comparison used a cylindrical hologram with a radius of $11 \mathrm{~mm}$, a height of $4 \mathrm{~mm}$, a sound speed of $2450 \mathrm{~m} \mathrm{~s}^{-1}$, and a density of $1180 \mathrm{~kg} \mathrm{~m}^{-3}$. A layer $2 \mathrm{~mm}$ below the top surface was used for the comparison. The target point was placed 1.8 $\mathrm{cm}$ above the cylinder displaced $2 \mathrm{~mm}$ from its centre in both the $\mathrm{x}$ and $\mathrm{y}$ directions. The ray-tracing algorithm was run for these inputs and compared against a k-Wave simulation.

The k-Wave simulation was carried out on a $256 \times 256 \times 288$ domain with a grid spacing of $0.1 \mathrm{~mm}$. The hologram was inserted at one end of the domain as a cylinder of matching dimensions, with the acoustic properties matching those of the hologram. The rest of the medium was set to a sound speed of $1500 \mathrm{~m} \mathrm{~s}^{-1}$ and density $1000 \mathrm{~kg} \mathrm{~m}^{-3}$. The target point was inserted as a source point 180 grid points above the cylinder, displaced by 20 grid points from its centre in $x$ and $y$. The pressure was recorded across a plane $2 \mathrm{~mm}$ into the hologram, to compare against the ray-tracing output. A bipolar pulse filtered at $6 \mathrm{MHz}$ was used as $s(t)$ in both the simulation and the ray-tracing. A comparison of the ray-tracing and $\mathrm{k}$ Wave outputs from 3 separate positions are shown in Fig. 2. The two show good agreement over the first $4 \mu \mathrm{s}$. This is sufficient for calculating the patterns.

\section{Pattern Calculation}

Once the impulse responses for each point have been calculated, they are used to calculate the binary pattern for each layer. The goal is to find the binary pattern $H(x, y, d)$ 
that will generate the greatest peak pressure $\max (p(\mathbf{r}, t))$ at each of the target points. This is subject to the constraint that for each $(x, y)$ position only a single pixel may be active over each of the layers. This is because the incident light will be mostly absorbed at the first pixel it interacts with.

The binary patterns are chosen using an optimisation approach based on direct binary search. This was based on a modification of an algorithm described in an earlier work [3]. This searches, by changing the state of individual pixels, for a pattern which minimises a cost function that penalises variations in peak pressure across the target points and rewards increases in peak pressure.

\section{EXPERIMENTS}

\section{A. Overview}

To validate that the proposed 3-D holograms could be used to generate patterned acoustic fields. Measurements were made of the acoustic field generated from fabricated samples, and numerical experiments were carried out using the k-Wave toolbox.

The dimensions of the hologram used were $50 \times 30 \times 6 \mathrm{~mm}$ with a pattern aperture of $20 \times 20 \mathrm{~mm}$ centred on the middle of the hologram. It had 3 distinct absorbing layers spaced every $2 \mathrm{~mm}$, with the first layer occurring on top. The hologram was composed of PMMA, which was taken to have a sound speed of $2750 \mathrm{~m} \mathrm{~s}^{-1}$ and a density of $1190 \mathrm{~kg} \mathrm{~m}^{-3}$ [10].

The hologram was designed to focus over a $3 \times 3$ array of points. These were evenly spaced with a separation of $3 \mathrm{~mm}$. This array was located in a plane parallel to the hologram $18 \mathrm{~mm}$ away and was coaligned with the central axis of the hologram. The approach described in Sec. II for hologram design was used to calculate the pattern for each layer. The signal generated from the absorber $s(t)$ was taken to be a broadband bipolar pulse filtered at $5 \mathrm{MHz}$. The resulting set of patterns are shown in Fig. 3.

\section{B. Hologram Fabrication}

The hologram was fabricated from a set of three $50 \times 30 \times 2$ mm transparent PMMA slides. These were each coated with a thin layer of black spray paint (Super Satin, Plasti Kote, Valspar, US). A laser cutter (VLS4.60, Universal Laser Systems, US) was used to etch the appropriate pattern from each absorbing layer. These absorbers were glued (Serious Glue, Bostik, Wisconsin, US) together to create the layered absorber. A photograph of the fabricated hologram is shown in Fig. 4(a).

\section{Field Measurements}

A fibre coupled Q-switch Nd:YAG laser (Ultra, Big Sky Laser Technologies, Bozeman MT) was used to excite ultrasound from the fabricated hologram. This had a wavelength of $1064 \mathrm{~nm}$, a pulse length of $8 \mathrm{~ns}$, a repetition rate of $20 \mathrm{~Hz}$ and pulse energies of $50 \mathrm{~mJ}$. Measurements of the acoustic field were made using a previously described optical scanner [11]. This uses a planar Fabry-Perot thin film interferometer for acoustic detection over a 2-D plane.
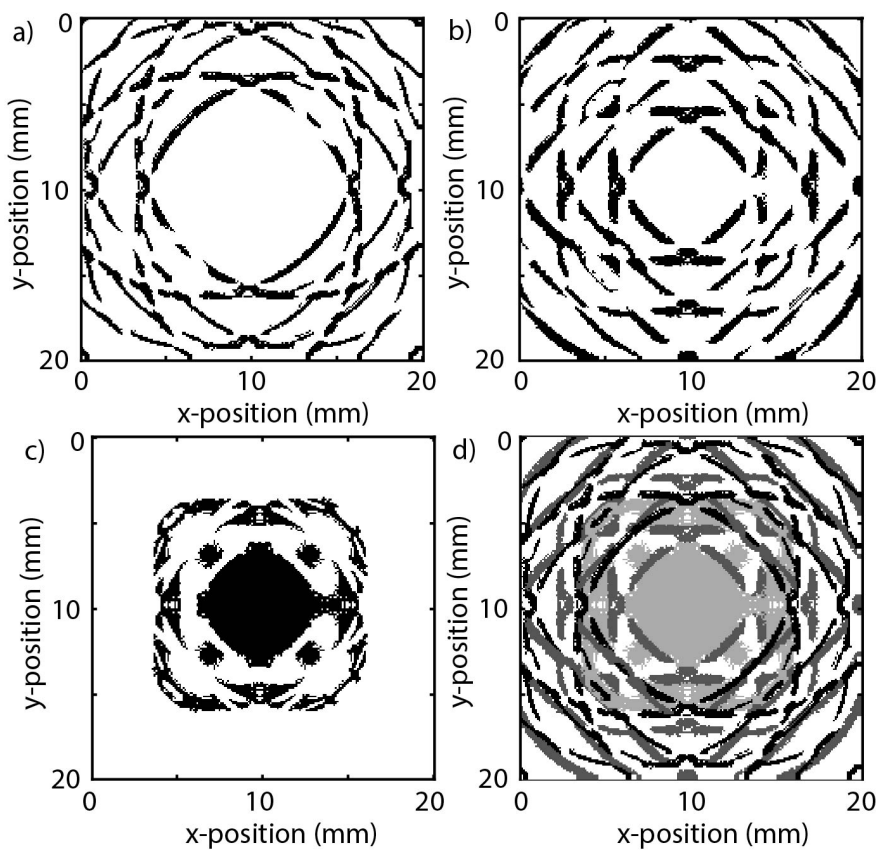

Fig. 3. (a) First layer of calculated hologram. (b) Second layer of calculated hologram. (c) Third layer of calculated hologram. (d) Composite of calculated hologram, different layers represented by different shades. The lack of overlap can be seen.

A photo of the experimental set up can be seen in Fig. 4(b). The absorber was suspended in water $10 \mathrm{~mm}$ above the surface of the sensor and parallel to it. The laser fibre tip was then placed $20 \mathrm{~cm}$ above the absorber and manually aligned with its centre. The radius of the beam at the absorber surface was approximately $20 \mathrm{~mm}$. Signals were recorded over a $2.46 \times 1.99 \mathrm{~cm}$ area centred on the absorber using a step size of $0.15 \mathrm{~mm}$. Four averages were taken at each position.

To calculate the 3-D wave-field of the hologram from the planar measurements, k-Wave was used to both forward and back propagate the planar measurements by $25 \mathrm{~mm}$ assuming linear propagation. Prior to this the datasets were low pass filtered at $5 \mathrm{MHz}$, and high pass filtered at $300 \mathrm{kHz}$.

\section{Numerical Experiments}

The experimental results were compared against a simulation of the acoustic field generated by the same pattern. This simulation was carried out using k-Wave on a $540 \times 324 \times 324$ grid, with a grid spacing of $0.1 \mathrm{~mm}$. The medium for this simulation was inhomogeneous. The 3-D absorber was inserted at one end of the simulation domain as a $500 \times 300 \times 60$ region with sound speed $2750 \mathrm{~m} \mathrm{~s}^{-1}$ and density $1190 \mathrm{~kg} \mathrm{~m}^{-3}$. The rest of the medium had a sound speed of $1500 \mathrm{~m} \mathrm{~s}^{-1}$ and 1000 $\mathrm{kg} \mathrm{m}^{-3}$ to represent water immersion.

The three patterned layers were inserted as source masks over 3 simulations planes. The first was placed in a plane one grid point above the hologram, and the second and third were placed at 20 and 40 grid points within the hologram region. The pressure $s(t)$ generated from the absorber was represented by a broadband bipolar pulse filtered at $5 \mathrm{MHz}$. 
a)

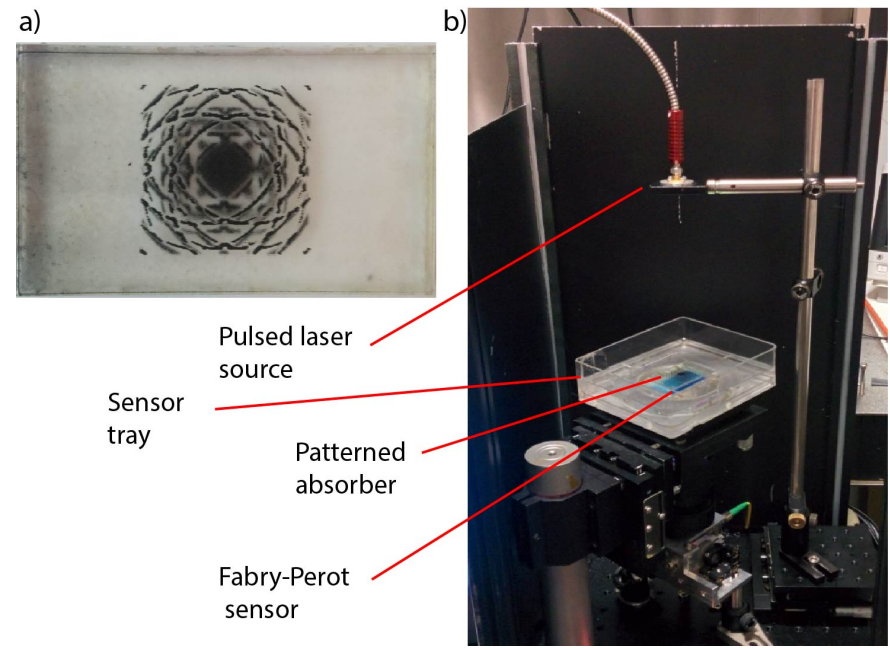

Fig. 4. (a) Photo of fabricated multi-layer absorber. (b) Annotated photo of experimental set-up.
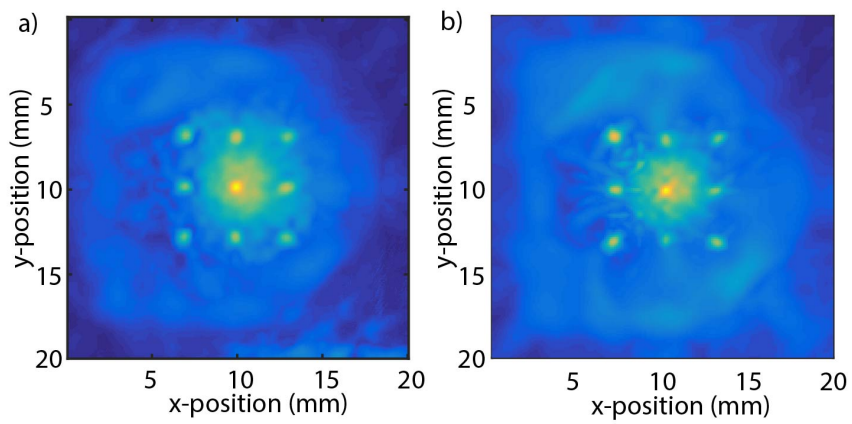

Fig. 5. (a) 9 foci generated in the target plane of the hologram in the experiment. (b) 9 foci generated in target plane of hologram in simulation correcting for apodisation.

In previous work it has been found that the spatial profile of the laser significantly effects the resulting acoustic field as it introduces an apodisation of the initial pressure distribution [3, 12]. A correction was applied for this. This was done by back propagating the measurements described in Sec. III C to $t=0$ to recover the initial pressure distribution. Each of the absorber planes were then identified in this initial pressure distribution and registered to the binary patterns displayed in Fig. 3(a)-(c). These spatial profiles were then used in the forward simulation for a better comparison with the experimental data.

\section{RESULTS}

The maximum pressure generated in the target plane of the multi-layer hologram in both the experimental and simulated data is shown in Fig. 5 (a) and (b). It can be seen that in both cases the targeted 9 foci are clearly realised and there is good qualitative agreement between the simulation and experiment. However, in both datasets the central focus is significantly more intense. This is due to the spatial profile of the laser, which is approximately Gaussian so introduces a greater weighting for the central focus. A high background can be seen surrounding the central focus. This is in part caused by the laser profile in addition to the low focal gain at the target points caused by the small number of absorbing layers.

\section{CONCLUSION}

This work has successfully demonstrated a new approach for the spatial control of optically generated acoustic fields. This uses multi-layer holograms to generate focused acoustic fields using a single optical pulse.

At present the realisation of the target patterns (signal to background) achieved using the ' 3 -D holograms' is poor. This is due to the low number of absorbing layers and the fixed layer spacing. These constrain the fraction of the aperture that can be used and lower the pressure contributed by each $(x, y)$ position. With a fabrication method capable of a narrower layer spacing, this could be overcome. This would enable the generation of complex, high pressure, optically generated acoustic fields from a single pulsed source.

\section{ACKNOWLEDGMENT}

The authors would like to thank the EPSRC for funding the work.

\section{REFERENCES}

[1] T. Buma, M. Spisar and M. O’Donnell, "High-frequency ultrasound array element using thermoelastic expansion in an elastomeric film", Appl. Phys. Lett, vol. 79, pp. 548-550, 2001.

[2] A. Meyer, S. Gspan, S. Bernet and M. Ritsch-Marte, "Tailoring ultrasonic beams with optoacoustic holography", Laser Resonators and Beam Control VI. Proc SPIE, vol. 4969, pp. 105-114, 2003.

[3] M. Brown, J. Jaros, B. Cox and B. Treeby, "Control of broadband optically generated ultrasound pulses using binary amplitude holograms", J. Acoust. Soc. Am, vol. 139, pp. 1637-1647, 2016.

[4] S. Gspan, A. Meyer, S. Bernet and M. Ritsch-Marte, "Synthetic acoustic holograms realised via optoacoustic methods", Photons plus Ultrasound: Imaging and Sensing, Proc. of SPIE, vol. 5697, pp. 99-106, 2005.

[5] H. Baac, J. Ok, A. Maxwell, K. Lee, Y. Chen, A. Hart, Z. Xu, E. Yoon and L. Guo, "Carbon-Nanotube Optoacoustic Lens for Focused Ultrasound Generation and High-Precision Targeted Therapy", Scientific Reports, vol. 2, Article: 989, 2012.

[6] K. Passler, R. Nuster, S. Gratt, P. Burgholzer and G. Paltauf, "Lasergeneration of ultrasonic X-waves using axicon transducers", Appl. Phys. Lett., vol. 94, 064108, 2009.

[7] S. Gspan, A. Meyer, S. Bernet, M. Ritsch-Marte, "Optoacoustic generation of a helicoidal ultrasonic beam", J. Acoust. Soc. Am., vol. 115, pp. 1142-1146, 2004.

[8] R. W. Young, "Sound Pressure in Water from Source in Air", J. Acoust. Soc. Am., vol. 50, pp. 1708-1716, 1971

[9] B. E. Treeby and B. T. Cox, "k-Wave: MATLAB toolbox for the simulation and reconstruction of photoacoustic wave-fields", J. Biomed. Opt., vol. 15, 021314, 2010.

[10] A. R. Selfridge, "Approximate Material Properties in Isotropic Materials", IEEE. Transactions on Sonics and Ultrasonics, vol. SU-32, no. 3 , pp. 381-394, 1985

[11] E. Zhang, J. Laufer, and P. Beard, "Backward-mode multiwavelength photoacoustic scanner using a planar Fabry Perot polymer film ultrasound sensor for high resolution three-dimensional imaging of biological tissues", Appl. Opt., vol. 47, no. 4, pp. 561-577, 2008.

[12] M. D. Brown, T. J. Allen, B. T. Cox, and B. E. Treeby, "Control of Optically Generated Ultrasound Fields Using Binary Amplitude Holograms", IEEE International Ultrasonics Symposium, pp. 1037-1040, 2014. 